DAMTP/97-76

\title{
COALGEBRA EXTENSIONS AND ALGEBRA COEXTENSIONS OF GALOIS TYPE
}

\author{
Tomasz Brzeziński卬 and Piotr M. Hajad ${ }^{2}$ \\ Department of Applied Mathematics and Theoretical Physics, \\ University of Cambridge, Silver St., Cambridge CB3 9EW, England.
}

\begin{abstract}
The notion of a coalgebra-Galois extension is defined as a natural generalisation of a Hopf-Galois extension. It is shown that any coalgebra-Galois extension induces a unique entwining map $\psi$ compatible with the right coaction. For the dual notion of an algebra-Galois coextension it is also proven that there always exists a unique entwining structure compatible with the right action.
\end{abstract}

\section{Introduction}

Hopf-Galois extensions can be viewed as non-commutative torsors or principal bundles with universal differential structure. From the latter point of view a quantum group gauge theory was introduced in [3] and developed in [7, 5]. It turns out that to develop gauge theory on quantum homogeneous spaces (e.g., the family of Podleś quantum spheres) or with braided groups (see [9] for a recent review), one needs to consider coalgebra bundles. Such a generalisation of gauge theory was proposed recently in [四]. It introduces the notions

\footnotetext{
${ }^{1}$ On leave from: Department of Theoretical Physics, University of Eódź, Pomorska 149/153, 90-236 Łódź, Poland. E-MAIL: TB121@AMTP.CAM.AC.UK

2 On leave from: Department of Mathematical Methods in Physics, Warsaw University, ul. Hoża 74, Warsaw, 00-682 Poland. E-MAIL: PMH33@AMTP.CAM.AC.UK
} 
of an entwining structure with an entwining map $\psi$, and a $\psi$-principal coalgebra bundle. The latter can be viewed as a generalisation of a Hopf-Galois extension, termed a $\psi$-Galois extension.

In the present paper we introduce the notion of a coalgebra-Galois extension as a natural generalisation of a Hopf-Galois extension. It is obtained by requiring that the coaction be left-linear over the coinvariants rather than an algebra map. We prove that such defined coalgebra-Galois extension induces a unique entwining map $\psi$ making the coalgebra-Galois extension $\psi$-Galois. We dualise coalgebra-Galois extensions and thus introduce the notion of an algebra-Galois coextension. We show that for such a coextension there exists an entwining map $\psi$ making it a $\psi$-Galois coextension (called dual $\psi$-principal coalgebra bundle in [4]). Finally, we prove that if two quotient coalgebras $C / I_{1}$ and $C / I_{2}$ cogenerate (in the sense of Definition 4.1) the coalgebra $C$ equipped with a group-like element, then the coinvariants of $C$ are the same as the intersection of the coinvariants of $C / I_{1}$ with the coinvariants of $C / I_{2}$.

Notation. Here and below $k$ denotes a field. All algebras are over $k$, associative and unital with the unit denoted by 1 . All algebra homomorphisms are assumed to be unital. We use the standard algebra and coalgebra notation, i.e., $\Delta$ is a coproduct, $m$ is a product, $\varepsilon$ is a counit, etc. The identity map from the space $V$ to itself is also denoted by $V$. The unadorned tensor product stands for the tensor product over $k$. For a $k$-algebra $A$ we denote by $\mathcal{M o d}_{A}$, ${ }_{A} \mathcal{M o d}$ and ${ }_{A} \operatorname{Mod}_{A}$ the category of right $A$-modules, left $A$-modules and $A$ bimodules respectively. Similarly, for a $k$-coalgebra $C$ we denote by $\mathcal{M o d}^{C}$, ${ }^{C} \mathcal{M o d}$ and ${ }^{C} \mathcal{M}$ od ${ }^{C}$ the category of right $C$-comodules, left $C$-comodules and $C$-bicomodules respectively. Also, by ${ }_{A} \mathcal{M o d}^{C}\left({ }^{C} \mathcal{M o d}_{A}\right)$ we denote the category of left (right) $A$-modules with the action ${ }_{V} \mu\left(\mu_{V}\right)$ and right (left) $C$ comodules with the coaction $\Delta_{V}\left({ }_{V} \Delta\right)$ such that $\Delta_{V}{ }^{\circ} V=\left({ }_{V} \mu \otimes C\right) \circ\left(A \otimes \Delta_{V}\right)$ $\left({ }_{V} \Delta \circ \mu_{V}=\left(C \otimes \mu_{V}\right) \circ\left({ }_{V} \Delta \otimes A\right)\right)$, i.e., $\Delta_{V}\left({ }_{V} \Delta\right)$ is right (left) $A$-linear. For coactions and coproducts we use Sweedler's notation with suppressed summation sign: $\Delta_{A}(a)=a_{(0)} \otimes a_{(1)}, \Delta(c)=c_{(1)} \otimes c_{(2)}$. 


\section{$2 \quad C$-Galois extensions}

First recall the definition of a Hopf-Galois extension (see [10 for a review).

Definition 2.1 Let $H$ be a Hopf algebra, $A$ be a right $H$-comodule algebra, and $B:=A^{c o H}:=\left\{a \in A \mid \Delta_{A} a=a \otimes 1\right\}$. We say that $A$ is a (right) HopfGalois extension (or $H$-Galois extension) of $B$ iff the canonical left A-module right $H$-comodule map

$$
\text { can }:=(m \otimes H) \circ\left(A \otimes_{B} \Delta_{A}\right): A \otimes_{B} A \longrightarrow A \otimes H
$$

is bijective.

Note that in the situation of Definition 2.1, both $A \otimes_{B} A$ and $A \otimes H$ are objects in ${ }_{A} \mathcal{M o d}^{H}$ via the maps $m \otimes_{B} A, A \otimes_{B} \Delta_{A}$ and $m \otimes A, A \otimes \Delta$ respectively. The canonical map can is a morphism in this category. Thus the extension $B \subseteq A$ is Hopf-Galois if $A \otimes_{B} A \cong A \otimes H$ as objects in ${ }_{A} \mathcal{M o d}^{H}$ by the canonical map can.

It has recently been observed in [2] that one can view quantum embeddable homogeneous spaces $B$ of a Hopf algebra $H$, such as the family (indexed by $[0, \infty])$ of quantum two-spheres of Podleś [11], as extensions by a coalgebra $C$. It is known (see p.200 in [11]) that, except for the North Pole sphere $(c=0)$, no other spheres of this family are quantum quotient spaces of $S U_{q}(2)$. They escape the standard Hopf-Galois description. To include this important case in Galois extension theory, one needs to generalise the notion of a Hopf-Galois extension to the case of an algebra extended to an algebra by a coalgebra (cf. [6] for the dual picture). This is obtained by weakening the requirement that $\Delta_{A}$ be an algebra map, and leads to the notion of a coalgebra-Galois extension. (A special case of this kind was considered in [14, p.291].)

Definition 2.2 Let $C$ be a coalgebra, $e \in C$ a group-like element, $A$ an algebra and a right $C$-comodule such that $\Delta_{A} 1=1 \otimes e$, and $B:=A_{e}^{\text {coC }}:=\{a \in$ $\left.A \mid \Delta_{A} a=a \otimes e\right\}$. We say that $A$ is a (right) coalgebra-Galois extension (or $C$-Galois extension) of $B$ iff 
(1) the right coaction $\Delta_{A}: A \longrightarrow A \otimes C$ is left linear over $B$ and

(2) the canonical left A-module right $C$-comodule map

$$
\text { can }:=(m \otimes C) \circ\left(A \otimes_{B} \Delta_{A}\right): A \otimes_{B} A \longrightarrow A \otimes C
$$

is bijective.

Note that the first condition ensures that $B$ is an algebra, so that the second condition makes sense. In what follows, we will consider only right coalgebraGalois extensions, and skip writing "right" for brevity. The conditions of Definition 2.2 suffice to make both $A \otimes_{B} A$ and $A \otimes C$ objects in ${ }_{A} \mathcal{M o d}{ }^{C}$ via the maps $m \otimes_{B} A, A \otimes_{B} \Delta_{A}$ and $m \otimes A, A \otimes \Delta$, respectively. The canonical map is again a morphism in ${ }_{A} \mathcal{M o d}^{C}$. The extension $B \subseteq A$ is $C$-Galois if can is an isomorphism in ${ }_{A} \mathcal{M o d}^{C}$.

By the reasoning as in the proof of Proposition 1.6 in [7], one can obtain an alternative (differential) definition of a coalgebra-Galois extension:

Proposition 2.3 Let $C$, e and $A$ be as above. Let $\Omega^{1} A:=$ Ker $m$ denote the universal calculus on $A$, and $C^{+}:=\operatorname{Ker} \varepsilon$ the augmentation ideal of $C$. The algebra $A$ is a $C$-Galois extension if and only if the following sequence of left A-modules is exact:

$$
0 \longrightarrow A\left(\Omega^{1} B\right) A \longrightarrow \Omega^{1} A \stackrel{\overline{c a n}}{\longrightarrow} A \otimes C^{+} \longrightarrow 0,
$$

where $\overline{c a n}:=(m \otimes C) \circ\left(A \otimes \Delta_{A}\right)$.

In the Hopf-Galois case, one can generalise the above sequence to a nonuniversal differential calculus in a straightforward manner:

$$
0 \longrightarrow A \Omega^{1}(B) A \longrightarrow \Omega^{1}(A) \stackrel{\tilde{\chi}}{\longrightarrow} A \otimes\left(H^{+} / R_{H}\right) \longrightarrow 0,
$$

where $R_{H}$ is the $a d_{R}$-invariant right ideal of the Hopf algebra $H$ defining a bicovariant calculus on $H$ [17], and $\tilde{\chi}$ is defined by a formula fully analogous to the formula for $\overline{c a n}$. Sequence (2.2) is a starting point of the quantumgroup gauge theory proposed in [3] and continued in [7]. The Hopf-Galois extension describes a quantum principal bundle with the universal differential 
calculus. Proposition 2.3 shows that the $C$-Galois extension can also be viewed as a generalisation of such a bundle, a principal coalgebra bundle. The theory of such bundles and connections on them (also for non-universal differential calculus) was developed in [4. More specifically, the theory considered in [4] uses the notion of an entwining structure (closely connected with the theory of factorisation of algebras considered in [8]) and identifies coalgebra principal bundles with $C$-Galois extensions constructed within this entwining structure.

The aim of this section is to show that to each $C$-Galois extension of Definition 2.2 there corresponds a natural entwining structure. Therefore the notions of a $C$-Galois extension of Definition 2.2 and a $\psi$-principal coalgebra bundle of [4, Proposition 2.2] are equivalent to each other. First we recall the definition of an entwining structure.

Definition 2.4 Let $C$ be a coalgebra, $A$ an algebra and let $\psi$ be a $k$-linear map $\psi: C \otimes A \rightarrow A \otimes C$ such that

$$
\begin{array}{ll}
\psi \circ(C \otimes m)=(m \otimes C) \circ(A \otimes \psi) \circ(\psi \otimes A), & \psi \circ(C \otimes \eta)=\eta \otimes C, \\
(A \otimes \Delta) \circ \psi=(\psi \otimes C) \circ(C \otimes \psi) \circ(\Delta \otimes A), & (A \otimes \varepsilon) \circ \psi=\varepsilon \otimes A,
\end{array}
$$

where $\eta$ is the unit map $\eta: \alpha \mapsto \alpha 1$. Then $C$ and $A$ are said to be entwined by $\psi$ and the triple $(A, C, \psi)$ is called an entwining structure.

Entwining structures can be also understood as follows. Given an algebra $A$ and a coalgebra $C$ we consider $A \otimes C$ as an object in ${ }_{A} \mathcal{M}$ od with the structure map $m \otimes C$. Similarly we consider $C \otimes A$ as an object in ${ }^{C} \mathcal{M}$ od via the map $\Delta \otimes A$. Then we have

Proposition 2.5 Let $\mu_{A \otimes C}: A \otimes C \otimes A \rightarrow A \otimes C$ and $\Delta_{C \otimes A}: C \otimes A \rightarrow$ $C \otimes A \otimes C$ be the maps making $A \otimes C$ an object in ${ }_{A} \mathcal{M o d}_{A}$ and $C \otimes A$ an object in ${ }^{C} \mathcal{M o d}^{C}$ correspondingly, and such that

$$
(\varepsilon \otimes A \otimes C) \circ \Delta_{C \otimes A}=\mu_{A \otimes C} \circ(\eta \otimes C \otimes A) .
$$

Then the pairs $\left(\mu_{A \otimes C}, \Delta_{C \otimes A}\right)$ of such compatible maps are in one-to-one correspondence with the entwining structures $(A, C, \psi)$. 
Proof. First assume that $C \otimes A \in{ }^{C} \mathcal{M o d}{ }^{C}$ and $A \otimes C \in{ }_{A} \mathcal{M o d}_{A}$. Then

$$
(\Delta \otimes A \otimes C) \circ \Delta_{C \otimes A}=\left(C \otimes \Delta_{C \otimes A}\right) \circ(\Delta \otimes A),
$$

and

$$
\mu_{A \otimes C} \circ(m \otimes C \otimes A)=(m \otimes C) \circ\left(A \otimes \mu_{A \otimes C}\right) .
$$

Define

$$
\psi=(\varepsilon \otimes A \otimes C) \circ \Delta_{C \otimes A}=\mu_{A \otimes C} \circ(\eta \otimes C \otimes A) .
$$

Then

$$
\begin{aligned}
(m \otimes C) & \circ(A \otimes \psi) \circ(\psi \otimes A) \\
& =(m \otimes C) \circ\left(A \otimes \mu_{A \otimes C}\right) \circ(A \otimes \eta \otimes C \otimes A) \circ(\psi \otimes A) \\
& =\mu_{A \otimes C} \circ(m \otimes C \otimes A) \circ(A \otimes \eta \otimes C \otimes A) \circ(\psi \otimes A) \\
& =\mu_{A \otimes C} \circ\left(\mu_{A \otimes C} \otimes A\right) \circ(\eta \otimes C \otimes A \otimes A) \\
& =\mu_{A \otimes C} \circ(A \otimes C \otimes m) \circ(\eta \otimes C \otimes A \otimes A) \\
& =\mu_{A \otimes C} \circ(\eta \otimes C \otimes A) \circ(C \otimes m)=\psi \circ(C \otimes m) .
\end{aligned}
$$

Furthermore

$$
\psi \circ(C \otimes \eta)=\mu_{A \otimes C} \circ(\eta \otimes C \otimes A) \circ(C \otimes \eta)=\mu_{A \otimes C} \circ(\eta \otimes C \otimes \eta)=\eta \otimes C .
$$

Therefore $\psi$ satisfies conditions (2.3). Dualising the above calculation (namely, interchanging $\Delta$ with $m, C$ with $A, \varepsilon$ with $\eta$ and $\Delta_{C \otimes A}$ with $\mu_{A \otimes C}$ ) one easily finds that $\psi$ satisfies conditions (2.4) too. Hence $(A, C, \psi)$ is an entwining structure.

Conversely, let $(A, C, \psi)$ be an entwining structure. Define $\Delta_{C \otimes A}=(C \otimes \psi) \circ$ $(\Delta \otimes A)$ and $\mu_{A \otimes C}=(m \otimes C) \circ(A \otimes \psi)$. Then

$$
\begin{aligned}
(C \otimes A \otimes \Delta) & \circ \Delta_{C \otimes A} \\
& =(C \otimes A \otimes \Delta) \circ(C \otimes \psi) \circ(\Delta \otimes A) \\
& =(C \otimes \psi \otimes C) \circ(C \otimes C \otimes \psi) \circ(C \otimes \Delta \otimes A) \circ(\Delta \otimes A) \\
& =(C \otimes \psi \otimes C) \circ(C \otimes C \otimes \psi) \circ(\Delta \otimes C \otimes A) \circ(\Delta \otimes A) \\
& =(C \otimes \psi \otimes C) \circ(\Delta \otimes A \otimes C) \circ(C \otimes \psi) \circ(\Delta \otimes A) \\
& =\left(\Delta_{C \otimes A} \otimes C\right) \circ \Delta_{C \otimes A} .
\end{aligned}
$$


We used property (2.4) to derive the second equality, and then coassociativity of the coproduct to derive the third one. Similarly,

$$
\begin{aligned}
(C \otimes A \otimes \varepsilon) \circ \Delta_{C \otimes A} & =(C \otimes A \otimes \varepsilon) \circ(C \otimes \psi) \circ(\Delta \otimes A) \\
& =(C \otimes \varepsilon \otimes A) \circ(\Delta \otimes A)=C \otimes A .
\end{aligned}
$$

Hence $\Delta_{C \otimes A}$ is a right coaction. By dualising the above argument one verifies that $\mu_{A \otimes C}$ is a right action. Finally, an elementary calculation shows that

$$
(\varepsilon \otimes A \otimes C) \circ \Delta_{C \otimes A}=\mu_{A \otimes C} \circ(\eta \otimes C \otimes A)=\psi .
$$

Thus the bijective correspondence is established.

As a consequence of the definition of an entwining structure one easily finds [4, Proposition 2.2] that for any group-like $e \in C$, we have $A \in \operatorname{Mod}^{C}$ with a coaction $\Delta_{A}: a \mapsto \psi(e \otimes a)$. Clearly $\Delta_{A}(1)=1 \otimes e$. Furthermore, for any $a \in A$ and any $b$ in the fixed-point subspace $B=A_{e}^{c o C} \equiv\left\{a \in A \mid \Delta_{A}(a)=\right.$ $a \otimes e\}$, we have

$$
\begin{aligned}
\Delta_{A}(b a) & =\psi(e \otimes b a)=((m \otimes C) \circ(A \otimes \psi))(\psi(e \otimes b) \otimes a) \\
& =((m \otimes C) \circ(A \otimes \psi))(b \otimes e \otimes a)=b \Delta_{A}(a) .
\end{aligned}
$$

Here we used the first of equations (2.3) to derive the second equality. Consequently, $B$ is an algebra, $\Delta_{A}$ is a left $B$-module map, $A \in{ }_{B} \mathcal{M o d}^{C}$ and we are in the setting of Definition 2.2. Now we can consider [ [ 1 , Proposition 2.2]:

Definition 2.6 Let $(A, C, \psi)$ be an entwining structure, $e \in C$ a group-like element, and $\Delta_{A}: a \mapsto \psi(e \otimes a)$ a right $C$-coaction on $A$. We say that $B \subseteq A$ is a $\psi$-Galois extension of $B=A_{e}^{c o C}$ by a coalgebra $C$ iff $B \subseteq A$ is a coalgebraGalois extension, i.e., iff the canonical homomorphism can : $A \otimes_{B} A \rightarrow A \otimes C$ in ${ }_{A} \mathcal{M o d}^{C}$ is an isomorphism. (We call the underlying quantum-space structure of $(A, B, C, \psi, e)$ a $\psi$-principal bundle. $)$

The main result of the paper is contained in the following:

Theorem 2.7 Let $A$ be a $C$-Galois extension of $B:=A_{e}^{c o C}$. Then there exists a unique map $\psi: C \otimes A \rightarrow A \otimes C$ entwining $C$ with $A$ and such that $\Delta_{A}(a)=$ $\psi(e \otimes a)$ for any $a \in A$. 
Proof. Assume that $B \subseteq A$ is a coalgebra-Galois extension. Then can is bijective and there exists the translation map $\tau: C \rightarrow A \otimes_{B} A, \tau(c):=$ $\operatorname{can}^{-1}(1 \otimes c)$. We use the notation $\tau(c)=c^{(1)} \otimes c^{(2)}$ (summation understood). Using [1, Proposition 3.9] or [13, Remark 3.4] and their obvious generalisation to the present case, for all $c \in C$ and $a \in A$, one obtains that

(i) $c^{(1)} c^{(2)}=\varepsilon(c)$,

(ii) $a_{(0)} a_{(1)}^{(1)} \otimes a_{(1)}^{(2)}=1 \otimes a$,

(iii) $c^{(1)} \otimes c^{(2)}{ }_{(0)} \otimes c^{(2)}{ }_{(1)}=c_{(1)}^{(1)} \otimes c_{(1)}^{(2)} \otimes c_{(2)}$.

Define a map $\psi: C \otimes A \rightarrow A \otimes C$ by

$$
\psi=\operatorname{can} \circ\left(A \otimes_{B} m\right) \circ(\tau \otimes A), \quad \psi(c \otimes a)=c^{(1)}\left(c^{(2)} a\right)_{(0)} \otimes\left(c^{(2)} a\right)_{(1)} .
$$

We show that $\psi$ entwines $C$ and $A$. By definition of the translation map, we have:

$$
\psi(c \otimes 1)=c^{(1)} c^{(2)}{ }_{(0)} \otimes c^{(2)}(1)=1 \otimes c .
$$

Furthermore, by property (i) of the translation map,

$$
((\mathrm{id} \otimes \varepsilon) \circ \psi)(c \otimes a)=c^{(1)}\left(c^{(2)} a\right)_{(0)} \otimes \varepsilon\left(\left(c^{(2)} a\right)_{(1)}\right)=c^{(1)} c^{(2)} a=\varepsilon(c) a .
$$

Thus we have proven that the second equations in (2.3)-(2.4) hold. Next we have

$$
\begin{aligned}
((m \otimes C) & \circ(A \otimes \psi) \circ(\psi \otimes A))\left(c \otimes a \otimes a^{\prime}\right) \\
& =((m \otimes C) \circ(A \otimes \psi))\left(c^{(1)}\left(c^{(2)} a\right)_{(0)} \otimes\left(c^{(2)} a\right)_{(1)} \otimes a^{\prime}\right) \\
& =c^{(1)}\left(c^{(2)} a\right)_{(0)}\left(c^{(2)} a\right)_{(1)}^{(1)}\left(\left(c^{(2)} a\right)_{(1)}^{(2)} a^{\prime}\right)_{(0)} \otimes\left(\left(c^{(2)} a\right)_{(1)}^{(2)} a^{\prime}\right)_{(1)} \\
& =c^{(1)}\left(c^{(2)} a a^{\prime}\right)_{(0)} \otimes\left(c^{(2)} a a^{\prime}\right)_{(1)}=(\psi \circ(C \otimes m))\left(c \otimes a \otimes a^{\prime}\right),
\end{aligned}
$$

where we used the property (ii) of the translation map to derive the third equality. Hence the first of equations (2.3) holds. Similarly,

$$
\begin{aligned}
((\psi \otimes C) \circ & (C \otimes \psi) \circ(\Delta \otimes A))(c \otimes a) \\
= & (\psi \otimes C)\left(c_{(1)} \otimes c_{(2)}{ }^{(1)}\left(c_{(2)}{ }^{(2)} a\right)_{(0)} \otimes\left(c^{(2)} a\right)_{(1)}\right) \\
= & c_{(1)}{ }^{(1)}\left(c_{(1)}{ }^{(2)} c_{(2)}{ }^{(1)}\left(c_{(2)}{ }^{(2)} a\right)_{(0)}\right)_{(0)} \otimes\left(c_{(1)}{ }^{(2)} c_{(2)}{ }^{(1)}\left(c_{(2)}{ }^{(2)} a\right)_{(0)}\right)_{(1)} \\
& \otimes\left(c_{(2)}{ }^{(2)} a\right)_{(1)}
\end{aligned}
$$




$$
\begin{aligned}
= & c^{(1)}\left(c^{(2)}{ }_{(0)} c^{(2)}{ }_{(1)}{ }^{(1)}\left(c^{(2)}{ }_{(1)}{ }^{(2)} a\right)_{(0)}\right)_{(0)} \otimes\left(c^{(2)}{ }_{(0)} c^{(2)}{ }_{(1)}{ }^{(1)}\left(c^{(2)}{ }_{(1)}{ }^{(2)} a\right)_{(0)}\right)_{(1)} \\
& \otimes\left(c^{(2)}{ }_{(1)}^{(2)} a\right)_{(1)} \\
= & c^{(1)}\left(\left(c^{(2)} a\right)_{(0)}\right)_{(0)} \otimes\left(\left(c^{(2)} a\right)_{(0)}\right)_{(1)} \otimes\left(c^{(2)} a\right)_{(1)} \\
= & c^{(1)}\left(c^{(2)} a\right)_{(0)} \otimes\left(c^{(2)} a\right)_{(1)} \otimes\left(c^{(2)} a\right)_{(2)}=((A \otimes \Delta) \circ \psi)(c \otimes a) .
\end{aligned}
$$

We used property (iii) of the translation map to derive the third equality and then property (ii) to derive the fourth one. Hence $C$ and $A$ are entwined by $\psi$ as required. Moreover, since $\tau(e)=1 \otimes 1$, we have

$$
\psi(e \otimes a)=e^{(1)}\left(e^{(2)} a\right)_{(0)} \otimes\left(e^{(2)} a\right)_{(1)}=a_{(0)} \otimes a_{(1)}=\Delta_{A}(a) .
$$

It remains to prove the uniqueness of the entwining map $\psi$ given by (2.5). Suppose that there is an entwining map $\tilde{\psi}$ such that $\tilde{\psi}(e \otimes a)=a_{(0)} \otimes a_{(1)}$, $a \in A$. Then, for all $a \in A, c \in C$,

$$
\begin{aligned}
\psi(c \otimes a) & =c^{(1)}\left(c^{(2)} a\right)_{(0)} \otimes\left(c^{(2)} a\right)_{(1)} \\
& =c^{(1)}(\tilde{\psi} \circ(C \otimes m))\left(e \otimes c^{(2)} \otimes a\right) \\
& =c^{(1)}((m \otimes C) \circ(A \otimes \tilde{\psi}) \circ(\tilde{\psi} \otimes A))\left(e \otimes c^{(2)} \otimes a\right) \\
& =c^{(1)}((m \otimes C) \circ(A \otimes \tilde{\psi}))\left(c^{(1)}{ }_{(0)} \otimes c^{(2)}{ }_{(1)} \otimes a\right) \\
& =c^{(1)} c_{(0)}^{(2)} \tilde{\psi}\left(c^{(2)}{ }_{(1)} \otimes a\right)=\tilde{\psi}(c \otimes a) .
\end{aligned}
$$

Here we used first of equations (2.3) to derive the third equality and then the definition of the translation map to obtain the last one. Thus the uniqueness of $\psi$ is proven and the proof of the theorem is completed.

The map $a \otimes_{B} a^{\prime} \mapsto a \psi\left(e \otimes a^{\prime}\right)$ coincides with the isomorphism can, whence $(A, B, C, \psi, e)$ is a $\psi$-Galois extension. We have:

Corollary 2.8 For a given algebra $A$, a coalgebra $C$, and a group-like $e \in C$, the following statements are equivalent:

(1) $(A, B, C, \psi, e)$ is a $\psi$-Galois extension.

(2) $B \subseteq A$ is a coalgebra-Galois extension of $B=A_{e}^{c o C}$ by $C$. 
Remark 2.9 A slightly different definition of a $C$-Galois extension was proposed in 2]. Let $A$ be an algebra, $C$ a coalgebra and $e$ a group-like element of $C$. One assumes that $A \otimes C \in \operatorname{Mod}_{A}, A \in \operatorname{Mod}^{C}$, and the action and coaction are such that $\Delta_{A} \circ m=\mu_{A \otimes C} \circ\left(\Delta_{A} \otimes A\right)$ and $\mu_{A \otimes C}\left(a \otimes e, a^{\prime}\right)=$ $a a_{(0)}^{\prime} \otimes a_{(1)}^{\prime}$ for any $a, a^{\prime} \in A$. Then $B=A_{e}^{c o C}$ is an algebra and the canonical map can : $A \otimes_{B} A \rightarrow A \otimes C$ is well-defined. $B \subseteq A$ is a $C$-Galois extension if the canonical map can is a bijection. One easily finds, however, that given $A$ and $C$ satisfying the above conditions, $A \in{ }_{B} \mathcal{M o d}^{C}$ via the maps $\mu_{A}=m$ and $\Delta_{A}$. Hence, by Theorem 2.7, this definition of a $C$-Galois extension is equivalent to the one introduced in [4] and in Definition 2.2.

Remark 2.10 In the Hopf-Galois case, the formula for $\psi$ becomes quite simple: $\psi(h \otimes a)=a_{(0)} \otimes h a_{(1)}$. If the Hopf algebra $H$ has a bijective antipode, $\psi$ is an isomorphism, and its inverse is given by $\psi^{-1}(a \otimes h):=h S^{-1}\left(a_{(1)}\right) \otimes a_{(0)}$. Furthermore, the coaction ${ }_{A} \Delta: A \rightarrow H^{o p} \otimes A$,

$$
{ }_{A} \Delta(a):=a_{(-1)} \otimes a_{(0)}:=S^{-1}\left(a_{(1)}\right) \otimes a_{(0)}
$$

makes $A$ a left $H^{o p}$-comodule algebra, where $H^{o p}$ stands for the Hopf algebra with the opposite multiplication. One can define the following left version of the canonical map: $\operatorname{can}_{L}: A \otimes_{B} A \rightarrow H \otimes A, \operatorname{can}_{L}\left(a \otimes_{B} a^{\prime}\right):=a_{(-1)} \otimes a_{(0)} a^{\prime}$. It is straightforward to verify that $\psi \circ \operatorname{can}_{L}=$ can. Since $\psi$ and can are isomorphisms, we can immediately conclude that so is $c a n_{L}$.

\section{$3 \quad$ A-Galois coextensions}

The dual version of a Hopf-Galois extension can be viewed as a non-commutative generalisation of the theory of quotients of formal schemes under free actions of formal group schemes (cf. 12]). In this section we dualise $C$-Galois extensions and derive results analogous to the results discussed in the previous section. First recall the definition of a cotensor product. Let $B$ be a coalgebra and $M$, $N$ a right and left $B$-comodule respectively. The cotensor product $M \square_{B} N$ is defined by the exact sequence

$$
0 \rightarrow M \square_{B} N \hookrightarrow M \otimes N \stackrel{\ell}{\rightarrow} M \otimes B \otimes N
$$


where $\ell$ is the coaction equalising map $\ell=\Delta_{M} \otimes N-M \otimes{ }_{N} \Delta$. In particular, if $C$ is a coalgebra, $I$ its coideal and $B=C / I$, then

$$
C \square_{B} C=\left\{\sum_{i} c^{i} \otimes \tilde{c}^{i} \in C \otimes C \mid \sum_{i} c_{(1)}^{i} \otimes \pi\left(c_{(2)}^{i}\right) \otimes \tilde{c}^{i}=\sum_{i} c^{i} \otimes \pi\left(\tilde{c}_{(1)}^{i}\right) \otimes \tilde{c}_{(2)}^{i}\right\},
$$

where $\pi: C \rightarrow B=C / I$ is the canonical surjection. The following definition [15, p.3346] dualises the concept of a Hopf-Galois extension:

Definition 3.1 Let $H$ be a Hopf algebra, $C$ a right $H$-module coalgebra with the action $\mu_{C}: C \otimes H \rightarrow C$. Then $I:=\left\{\mu_{C}(c, h)-\varepsilon(h) c \mid c \in C, h \in H\right\}$ is a coideal in $C$ and thus $B:=C / I$ is a coalgebra. We say that $C \rightarrow B$ is a Hopf-Galois coextension (or H-Galois coextension) iff the canonical left $\mathrm{C}$-comodule right $\mathrm{H}$-module map

$$
\text { cocan }:=\left(C \otimes \mu_{C}\right) \circ(\Delta \otimes H): C \otimes H \longrightarrow C \square_{B} C
$$

is a bijection.

With the help of the property $\Delta \circ \mu_{C}=\left(\mu_{C} \otimes \mu_{C}\right) \circ(C \otimes$ flip $\otimes H) \circ(\Delta \otimes \Delta)$, it can be directly checked that the image of the map cocan is indeed contained in $C \square_{B} C$. To see more clearly that Definition 3.1 is obtained by dualising Definition 2.1, one can notice that both $C \otimes H$ and $C \square_{B} C$ are objects in ${ }^{C} \operatorname{Mod}_{H}$, which is dual to ${ }_{A} \mathcal{M ~ M o d ~}^{H}$. The structure maps are $\Delta \otimes C, C \otimes m$ and $\Delta \square_{B} C, C \square_{B} \mu_{C}$ respectively. The canonical map cocan is a morphism in ${ }^{C} \mathcal{M o d}_{H}$. The coextension $C \rightarrow B$ is Hopf-Galois if $C \otimes H \cong C \square_{B} C$ as objects in ${ }^{C} \mathcal{M o d}_{H}$ by the canonical map cocan.

The notion of a Hopf-Galois coextension can be generalised by replacing $H$ by an algebra $A$ and weakening the condition that the action $\mu_{C}$ is a coalgebra map. This generalisation dualises the construction of a $C$-Galois extension of the previous section. First we prove

Lemma 3.2 Let $C$ be a coalgebra, $A$ an algebra and $\kappa: A \rightarrow k$ an algebra character. Assume that $C \in \operatorname{Mod}_{A}$ with the action $\mu_{C}$ and define

$$
I_{\kappa}=\left\{\mu_{C}(c, a)-\kappa(a) c \mid c \in C, a \in A\right\} .
$$


Let $B:=C / I_{\kappa}$. If $\varepsilon \circ \mu_{C}=\varepsilon \otimes \kappa$ and $\Delta_{\kappa} \circ \mu_{C}=\left(B \otimes \mu_{C}\right) \circ\left(\Delta_{\kappa} \otimes A\right)$, where $\Delta_{\kappa}=\left(\pi_{\kappa} \otimes C\right) \circ \Delta$ and $\pi_{\kappa}: C \rightarrow C / I_{\kappa}$ is the canonical surjection, then $B$ is a coalgebra. (If the conditions of the lemma are satisfied we say that the action $\mu_{C}$ is left $B$-colinear and counital with respect to $\kappa$.)

Proof. By the counitality of $\mu_{C}$, we have $I_{\kappa} \subseteq C^{+}:=\operatorname{Ker} \varepsilon$. Furthermore, using the colinearity of $\mu_{C}$ one finds that $\Delta_{\kappa}\left(I_{\kappa}\right) \subseteq B \otimes I_{\kappa}$. Since Ker $\pi_{\kappa}=I_{\kappa}$, we obtain $\Delta\left(I_{\kappa}\right) \subseteq C \otimes I_{\kappa}+I_{\kappa} \otimes C$. Therefore $I_{\kappa}$ is a coideal and $B$ is a coalgebra, as stated.

The above lemma implies that in this case $C \in{ }^{B} \mathcal{M} \operatorname{dod}_{A}$ with the structure maps $\mu_{C}$ and $\Delta_{\kappa}$. Consequently, the canonical map

$$
\operatorname{cocan}:=\left(C \otimes \mu_{C}\right) \circ(\Delta \otimes A): C \otimes A \longrightarrow C \square_{B} C
$$

is well defined, and we can consider:

Definition 3.3 Let $A$ be an algebra, $\kappa: A \rightarrow k$ an algebra character, $C$ a coalgebra and right $A$-module, and $B=C / I_{\kappa}$, where $I_{\kappa}$ is the coideal (3.6). We say that $C$ is an algebra-Galois coextension (or A-Galois coextension) of $B$ iff

(1) the right action $\mu_{C}: C \otimes A \rightarrow C$ is left colinear over $B$ and counital with respect to $\kappa$,

(2) the canonical left $C$-comodule right $A$-module map

$$
\text { cocan }:=\left(C \otimes \mu_{C}\right) \circ(\Delta \otimes A): C \otimes A \longrightarrow C \square_{B} C
$$

is bijective.

Again, to see that Definition 3.3 dualises the notion of a $C$-Galois extension one can notice that both $C \otimes A$ and $C \square_{B} C$ are objects in ${ }^{C} \mathcal{M o d} A$, which is dual to ${ }_{A} \mathcal{M o d}^{C}$. The structure maps are $\Delta \otimes C, C \otimes m$ and $\Delta \square_{B} C, C \square_{B} \mu_{C}$, respectively. The canonical map cocan is a morphism in ${ }^{C} \mathcal{M o d}{ }_{A}$. The coextension $C \rightarrow B$ is $A$-Galois if $C \otimes A \cong C \square_{B} C$ as objects in ${ }^{C} \mathcal{M o d}_{A}$ by the canonical map cocan. 
In [4] the notion of a dual $\psi$-principal bundle was introduced in the framework of entwining structures. This was possible by noting that the notion of an entwining structure has the following self-duality property. If one replaces $A$ with $C, \Delta$ with $m, \eta$ with $\varepsilon$ and reverses the order of composition, then formulae (2.3) become (2.4) and vice versa. Furthermore, if there is an algebra character $\kappa: A \rightarrow k$ one can define a right action $\mu_{C}: C \otimes A \rightarrow C$ by $\mu_{C}=(\kappa \otimes C) \circ \psi$. This action is counital with respect to $\kappa$. It is also left colinear over $B:=C / I_{\kappa}$, where $I_{\kappa}$ is given by (3.6). Indeed,

$$
\begin{aligned}
\Delta_{\kappa} \circ \mu_{C} & =\left(\pi_{\kappa} \otimes C\right) \circ \Delta \circ(\kappa \otimes C) \circ \psi \\
& =\left(\pi_{\kappa} \otimes C\right) \circ(\kappa \otimes C \otimes C) \circ(A \otimes \Delta) \circ \psi \\
& =\left(\pi_{\kappa} \otimes C\right) \circ(\kappa \otimes C \otimes C) \circ(\psi \otimes C) \circ(C \otimes \psi) \circ(\Delta \otimes A) \\
& =\left(\pi_{\kappa} \circ \mu_{C} \otimes C\right) \circ(C \otimes \psi) \circ(\Delta \otimes A) \\
& =\left(\pi_{\kappa} \otimes \kappa \otimes A\right) \circ(C \otimes \psi) \circ(\Delta \otimes A) \\
& =\left(\pi_{\kappa} \otimes \mu_{C}\right) \circ(\Delta \otimes A) \\
& =\left(B \otimes \mu_{C}\right) \circ\left(\Delta_{\kappa} \otimes A\right) .
\end{aligned}
$$

We used the definition of $\mu_{C}$ to derive the first, fourth and sixth equalities, the definition of $I_{\kappa}$ to derive the fifth one, and the first of equations (2.4) to establish the third equality. By Lemma $3.2 B$ is a coalgebra, and we can consider algebra-Galois coextensions whose right action map $\mu_{C}$ is determined by $\psi$ as described above.

Definition 3.4 Let $(A, C, \psi)$ be an entwining structure, $\kappa: A \rightarrow k$ an algebra character, and $\mu_{C}:=(\kappa \otimes C) \circ \psi$ a right action of $A$ on $C$. We say that $C \rightarrow B$ is a $\psi$-Galois coextension of $B=C / I_{\kappa}$ by an algebra $A$ iff $C \rightarrow B$ is an $A$ Galois coextension, i.e., iff the canonical morphism cocan : $C \otimes A \rightarrow C \square_{B} C$ in ${ }^{C} \mathcal{M o d}_{A}$ is an isomorphism. (We call the underlying quantum-space structure of $(A, B, C, \psi, \kappa)$ a dual $\psi$-principal bundle.)

Very much as in the previous section, it turns out that every $A$-Galois extension is equipped with an entwining structure. More precisely, we have the following dual version of Theorem 2.7: 
Theorem 3.5 Let $C$ be an $A$-Galois coextension of $B:=C / I_{\kappa}$. Then there exists a unique map $\psi: C \otimes A \rightarrow A \otimes C$ entwining $C$ with $A$ and such that $\mu_{C}=(\kappa \otimes C) \circ \psi$.

Proof. We dualise the proof of Theorem 2.7. Assume that $C \rightarrow B$ is an $A$ Galois coextension. Then cocan is a bijection and there exists the cotranslation map $\check{\tau}: C \square_{B} C \rightarrow A, \check{\tau}:=(\varepsilon \otimes C) \circ$ cocan $^{-1}$. By dualising properties of the translation map (or directly from the definition of $\check{\tau}$ ), one can establish the following properties of the cotranslation map:

(i) $\check{\tau}\left(c_{(1)}, c_{(2)}\right)=\varepsilon(c)$,

(ii) $\mu_{C} \circ(C \otimes \check{\tau}) \circ\left(\Delta \square_{B} C\right)=\varepsilon \otimes C$ or explicitly $\mu_{C}\left(c_{(1)}, \check{\tau}\left(c_{(2)}, c^{\prime}\right)=\varepsilon(c) c^{\prime}\right.$, (iii) $\check{\tau}\left(c, \mu_{C}\left(c^{\prime}, a\right)\right)=\check{\tau}\left(c, c^{\prime}\right) a$,

for any $c, c^{\prime} \in C$ and $a \in A$. Combining (iii) with (ii) one obtains

(iv) $\check{\tau}\left(c, c_{(1)}^{\prime}\right) \check{\tau}\left(c_{(2)}^{\prime}, c^{\prime \prime}\right)=\varepsilon\left(c^{\prime}\right) \check{\tau}\left(c, c^{\prime \prime}\right)$, for any $c, c^{\prime}, c^{\prime \prime} \in C$.

Using the cotranslation map one defines a map $\psi: C \otimes A \rightarrow A \otimes C$ by

$$
\begin{aligned}
& \psi=(\check{\tau} \otimes C) \circ(C \otimes \Delta) \circ \text { cocan, } \\
& \psi(c \otimes a)=\check{\tau}\left(c_{(1)}, \mu_{C}\left(c_{(2)}, a\right)_{(1)}\right) \otimes \mu_{C}\left(c_{(2)}, a\right)_{(2)} .
\end{aligned}
$$

We now show that the map $\psi$ entwines $C$ and $A$. For any $c \in C$ we find

$$
\psi(c \otimes 1)=\check{\tau}\left(c_{(1)}, \mu_{C}\left(c_{(2)}, 1\right)_{(1)}\right) \otimes \mu_{C}\left(c_{(2)}, 1\right)_{(2)}=\check{\tau}\left(c_{(1)}, c_{(2)}\right) \otimes c_{(3)}=1 \otimes c,
$$

where we used property (i) to derive the last equality. Furthermore, for any $c \in C, a \in A$,

$$
\begin{aligned}
((A \otimes \varepsilon) \circ \psi)(c \otimes a) & =\check{\tau}\left(c_{(1)}, \mu_{C}\left(c_{(2)}, a\right)_{(1)}\right) \varepsilon\left(\mu_{C}\left(c_{(2)}, a\right)_{(2)}\right) \\
& =\check{\tau}\left(c_{(1)}, \mu_{C}\left(c_{(2)}, a\right)\right)=\check{\tau}\left(c_{(1)}, c_{(2)}\right) a=\varepsilon(c) a
\end{aligned}
$$

where the penultimate equality is implied by (iii), and the ultimate one by (i). Thus we have proven that the second conditions of (2.3) and (2.4) are fulfilled by $\psi$. To prove the first of equations (2.3), we take $a, a^{\prime} \in A, c \in C$ and 
compute

$$
\begin{aligned}
&((m \otimes C) \circ(A \otimes \psi) \circ(\psi \otimes A))\left(c \otimes a \otimes a^{\prime}\right) \\
&=((m \otimes C) \circ(A \otimes \psi))\left(\check{\tau}\left(c_{(1)}, \mu_{C}\left(c_{(2)}, a\right)_{(1)}\right) \otimes \mu_{C}\left(c_{(2)}, a\right)_{(2)} \otimes a^{\prime}\right) \\
&=\check{\tau}\left(c_{(1)}, \mu_{C}\left(c_{(2)}, a\right)_{(1)}\right) \check{\tau}\left(\mu_{C}\left(c_{(2)}, a\right)_{(2)}, \mu_{C}\left(\mu_{C}\left(c_{(2)}, a\right)_{(3)}, a^{\prime}\right)_{(1)}\right) \\
& \otimes \mu_{C}\left(\mu_{C}\left(c_{(2)}, a\right)_{(3)}, a^{\prime}\right)_{(2)} \\
&= \check{\tau}\left(c_{(1)}, \mu_{C}\left(\mu_{C}\left(c_{(2)}, a\right), a^{\prime}\right)_{(1)}\right) \otimes \mu_{C}\left(\mu_{C}\left(c_{(2)}, a\right), a^{\prime}\right)_{(2)} \\
&= \check{\tau}\left(c_{(1)}, \mu_{C}\left(c_{(2)}, a a^{\prime}\right)_{(1)}\right) \otimes \mu_{C}\left(c_{(2)}, a a^{\prime}\right)_{(2)}=\psi\left(c \otimes a a^{\prime}\right) .
\end{aligned}
$$

We used property (iv) of the cotranslation map to derive the third equality. The fourth equality follows from the fact that $\mu_{C}$ is a right action. To prove the first of equations (2.4) we take any $c \in C, a \in A$, use (ii) and compute

$$
\begin{aligned}
&((\psi \otimes C) \circ(C \otimes \psi) \circ(\Delta \otimes A))(c \otimes a) \\
&=(\psi \otimes C)\left(c_{(1)} \otimes \check{\tau}\left(c_{(2)}, \mu_{C}\left(c_{(3)}, a\right)_{(1)}\right) \otimes \mu_{C}\left(c_{(3)}, a\right)_{(2)}\right) \\
&=\quad \check{\tau}\left(c_{(1)}, \mu_{C}\left(c_{(2)}, \check{\tau}\left(c_{(3)}, \mu_{C}\left(c_{(4)}, a\right)_{(1)}\right)\right)_{(1)}\right) \\
& \otimes \mu_{C}\left(c_{(2)}, \check{\tau}\left(c_{(3)}, \mu_{C}\left(c_{(4)}, a\right)_{(1)}\right)\right)_{(2)} \otimes \mu_{C}\left(c_{(4)}, a\right)_{(2)} \\
&= \check{\tau}\left(c_{(1)}, \mu_{C}\left(c_{(2)}, a\right)_{(1)}\right) \otimes \mu_{C}\left(c_{(2)}, a\right)_{(2)} \otimes \mu_{C}\left(c_{(2)}, a\right)_{(3)} \\
&=((A \otimes \Delta) \circ \psi)(c \otimes a) .
\end{aligned}
$$

Next observe that, by the counitality of $\mu_{C}$, we have $(\varepsilon \otimes \varepsilon) \circ$ cocan $=$ $(\varepsilon \otimes \kappa)$, whence $\kappa \circ \check{\tau}=\varepsilon \otimes \varepsilon$. This applied to the definition of $\psi$ implies that $(\kappa \otimes C) \circ \psi=\mu_{C}$, as needed. Finally, the uniqueness of $\psi$ can be verified by the dualisation of the argument at the end of the proof of Theorem 2.7. (To perform explicit calculations one needs to use the first of equations (2.4) and the property (iii) of the cotranslation map.)

As in the previous section, we have

Corollary 3.6 For an algebra $A$, a coalgebra $C$ and an algebra character $\kappa$ : $A \rightarrow k$, the following statements are equivalent:

(1) $(A, B, C, \psi, \kappa)$ is a $\psi$-Galois coextension.

(2) $C \rightarrow B$ is an $A$-Galois coextension of $B:=C / I_{\kappa}$ by $A$. 


\section{Appendix}

We say that two subgroups $G_{1}$ and $G_{2}$ of a group $G$ generate $G$ if any element of $G$ can be written as a finite length word whose letters are elements of $G_{1}$ or $G_{2}$. The (dual) coalgebra version of this concept is given in the following definition:

Definition 4.1 Let $C$ be a coalgebra and $I_{1}, I_{2}$ its coideals. Let $\wp_{(i)}$ denote the composite map

$$
C \stackrel{\Delta_{n}}{\longrightarrow} C^{\otimes n} \stackrel{\pi_{i_{1}} \otimes \ldots \otimes \pi_{i_{n}}}{\longrightarrow} C / I_{i_{1}} \otimes \ldots \otimes C / I_{i_{n}}
$$

where $\Delta_{n}(c):=c_{(1)} \otimes \ldots \otimes c_{(n)},(i):=\left(i_{1}, \ldots, i_{n}\right) \in\{1,2\}^{\times n}$ is a finite multiindex, and each $\pi_{i_{k}}$ is a canonical surjection. We say that the quotient coalgebras $C / I_{1}$ and $C / I_{2}$ cogenerate $C$ iff $\bigcap_{(i) \in \mathcal{M}_{f}} \operatorname{Ker} \wp_{(i)}=0$, where $\mathcal{M}_{f}$ is the space of all finite multi-indices. We write then $\left(C / I_{1}\right) \cdot\left(C / I_{2}\right)=C$.

Observe that the above construction is closely related to the wedge construction of 16. In the group situation it is clear that what is invariant under both generating subgroups is invariant under the whole group, and vice-versa. Below is the (dual) coalgebra version of this classical phenomenon.

Proposition 4.2 Let $C$ be a coalgebra, $e \in C$ a group-like element, $I_{1}$ and $I_{2}$ coideals of $C$, and $A$ a right $C$-comodule. Then

$$
\left(C / I_{1}\right) \cdot\left(C / I_{2}\right)=C \Rightarrow A_{e}^{c o C}=A_{e}^{c o\left(C / I_{1}\right)} \cap A_{e}^{c o\left(C / I_{2}\right)} .
$$

Proof. Clearly, we always have $A_{e}^{c o C} \subseteq A_{e}^{c o\left(C / I_{1}\right)} \cap A_{e}^{c o\left(C / I_{2}\right)}$. Assume now that there exists $a \in A_{e}^{c o\left(C / I_{1}\right)} \cap A_{e}^{c o\left(C / I_{2}\right)}$ such that $a \notin A_{e}^{c o C}$. Then $0 \neq \Delta_{A} a-a \otimes e=$ : $\sum_{j \in \mathcal{J}} f_{j} \otimes h_{j}$, where $\left\{f_{\alpha}\right\}_{\alpha \in \mathcal{A}}$ is a basis of $A$ and $\left\{h_{j}\right\}_{j \in \mathcal{J}}$ is a non-empty set which does not contain zero. Furthermore, for any $(i) \in \mathcal{M}_{f}$, we have:

$$
\begin{aligned}
& \left(A \otimes \wp_{(i)}\right)\left(\sum_{j \in \mathcal{J}} f_{j} \otimes h_{j}\right) \\
& =\left(\left(A \otimes \pi_{i_{1}} \otimes \cdots \otimes \pi_{i_{n}}\right) \circ\left(A \otimes \Delta_{n-1}\right)\right)\left(\Delta_{A} a-a \otimes e\right) \\
& =\left(\left(A \otimes \pi_{i_{1}} \otimes \cdots \otimes \pi_{i_{n}}\right) \circ\left(A \otimes \Delta_{n-1}\right) \circ \Delta_{A}\right)(a)-a \otimes e^{\otimes n}
\end{aligned}
$$




$$
\begin{aligned}
& =a_{(0)} \otimes \pi_{i_{1}}\left(a_{(1)}\right) \otimes \cdots \otimes \pi_{i_{n}}\left(a_{(n)}\right)-a \otimes e^{\otimes n} \\
& =\left(\left(A \otimes \pi_{i_{1}}\right) \circ \Delta_{A} \otimes C^{\otimes(n-1)}\right)\left(a_{(0)} \otimes \pi_{i_{2}}\left(a_{(1)}\right) \otimes \cdots \otimes \pi_{i_{n}}\left(a_{(n-1)}\right)\right)-a \otimes e^{\otimes n} \\
& =\left(\left[\left(A \otimes \pi_{i_{1}}\right) \circ \Delta_{A} \otimes C^{\otimes(n-1)}\right] \circ \cdots \circ\left(A \otimes \pi_{i_{n}}\right) \circ \Delta_{A}\right)(a)-a \otimes e^{\otimes n} \\
& =0
\end{aligned}
$$

Consequently, by the linear independence of $f_{j}, j \in \mathcal{J}$, we have $\wp_{(i)}\left(h_{j}\right)=0$ for $j \in \mathcal{J}$. Hence, as this is true for any $(i) \in \mathcal{M}_{f}$, we obtain $\bigcap_{(i) \in \mathcal{M}_{f}} \operatorname{Ker} \wp_{(i)} \neq 0$, as needed.

\section{ACKNOWLEDGEMENTS}

This work was supported by the EPSRC grant GR/K02244 (T.B.) and by the NATO postdoctoral fellowship and KBN grant 2 P301 02007 (P.M.H.).

\section{References}

[1] T. Brzeziński. Translation map in quantum principal bundles. J. Geom. Phys., 20:349-370, 1996. hep-th/9407145

[2] T. Brzeziński. Quantum homogeneous spaces as quantum quotient spaces. J. Math. Phys., 37:2388-2399, 1996. (q-alg/9509015)

[3] T. Brzeziński and S. Majid. Quantum group gauge theory on quantum spaces. Commun. Math. Phys., 157:591-638, 1993. Erratum 167:235, 1995. (hep-th/9208007)

[4] T. Brzeziński and S. Majid. Coalgebra bundles. To appear in Commun. Math. Phys. Preprint, q-alg/9602022, 1996.

[5] T. Brzeziński and S. Majid. Quantum differentials and the q-monopole revisited. Preprint DAMTP/97-60, q-alg/9706021, 1997.

[6] M. S. Dijkhuizen and T. Koornwinder. Quantum homogeneous spaces, duality and quantum 2-spheres. Geom. Dedicata, 52:291-315, 1994.

[7] P. M. Hajac. Strong connections on quantum principal bundles. Commun. Math. Phys. 182:579-617, 1996. 
[8] S. Majid. Physics for algebraists: Non-commutative and non-cocommutative Hopf algebras by a bicrossproduct construction. J. Algebra 130:1764, 1990.

[9] S. Majid. Advances in quantum and braided geometry. To appear in ed. V. Dobrev, Proc. XXI ICGTMP (Quantum groups volume), Goslar 1996, Heron Press, Sofia. (q-alg/9610003)

[10] S. Montgomery. Hopf Algebras and Their Actions on Rings. AMS, 1993.

[11] P. Podleś. Quantum spheres. Lett. Math. Phys., 14:193-202, 1987.

[12] H.-J. Schneider. Principal homogeneous spaces for arbitrary Hopf algebras. Isr. J. Math., 72:167-195, 1990.

[13] H.-J. Schneider. Representation theory of Hopf-Galois extensions. Isr. J. Math., 72:196-231, 1990.

[14] H.-J. Schneider. Normal basis and transitivity of crossed products for Hopf algebras. J. Algebra, 152:289-312, 1992.

[15] H.-J. Schneider. Some remarks on exact sequences of quantum groups. Comm. Alg., 21:3337-3357 (9), 1993

[16] M. E. Sweedler. Hopf Algebras W.A.Benjamin, Inc., New York, 1969

[17] S. L. Woronowicz. Differential calculus on compact matrix pseudogroups (quantum groups). Commun. Math. Phys. 122:125-170, 1989. 